\title{
Efecto del Mg y pH en la reducción de la toxicidad de Al en plantas de banano cultivadas en solución hidropónica
}

\section{Mg and pH effect on reducing Al toxicity in banana plants grown in hydroponic solution}

\author{
Hernán Sancho ${ }^{1}$, Eloy Molina ${ }^{2}$ \\ ${ }^{I}$ Consultor en Suelos, San José, Costa Rica. \\ ${ }^{2}$ Centro de Investigaciones Agronómicas, Universidad de Costa Rica, San José, Costa Rica.
}

\section{Resumen}

Se condujo un ensayo para evaluar la toxicidad del Al en el crecimiento y absorción de agua y nutrimentos en plantas de banano y el efecto del $\mathrm{Mg}$ y el $\mathrm{pH}$ en la toxicidad del Al. Los tratamientos fueron cuatro dosis de $\mathrm{Mg}(25,50,100$, $\left.200 \mathrm{mg}^{-1}\right)$ y dos dosis de $\mathrm{Al}\left(0\right.$ y $\left.25 \mathrm{mg} \cdot \mathrm{l}^{-1}\right)$ a tres diferentes valores de $\mathrm{pH}$ de manera que la combinación de dosis de Mg-Al y pH produjo los siguientes tratamientos 1) $25-0 \mathrm{pH} 4$; 2) $25-25 \mathrm{pH} 3$; 3) $50-25 \mathrm{pH} 3$; 4) 100-25 pH 3; 5) $200-25 \mathrm{pH} 3$; 6) $25-25 \mathrm{pH}$ 5. Se utilizaron plantas provenientes de cultivo de tejidos del clon "Gran Naine" que se pusieron a crecer por 63 días en solución nutritiva en potes de 201 de capacidad. Las variables evaluadas fueron: altura de planta, circunferencia de pseudo tallo, producción de biomasa, ritmo de emisión foliar, área foliar y absorción de nutrimentos. El Al redujo el valor de todas las variables de crecimiento con excepción del ritmo de emisión foliar. La dosis de $50 \mathrm{mg} \mathrm{l}^{-1}$ de $\mathrm{Mg}$ disminuyó parcialmente el efecto de la toxicidad del Al en la altura de planta y circunferencia de pseudo tallo. Dosis más altas de $\mathrm{Mg}$ presentaron resultados similares a $25 \mathrm{mg} \mathrm{l}^{-1}$. $\mathrm{El} \mathrm{Al}$ afectó la biomasa total, principalmente la de las raíces de primer orden, pero al subir el $\mathrm{Mg}$ disminuyó parcialmente este efecto. El Al disminuyó la absorción de agua, pero la dosis de $50 \mathrm{mg} \mathrm{l}^{-1}$ de $\mathrm{Mg}$ disminuyó levemente el efecto del $\mathrm{Al}$ en esta variable. La toxicidad de $\mathrm{Al}$ se redujo al aumentar el $\mathrm{pH}$ de 3 a 5 . El Al redujo la producción de biomasa en $65 \%$, mientras la absorción total de nutrimentos disminuyó entre 56 y $89 \%$. El orden de reducción del contenido de los macronutrimentos en los tejidos fue $\mathrm{Mg} \geq \mathrm{Ca}>\mathrm{S}>\mathrm{N} \geq \mathrm{K}>\mathrm{P}$ y el de los micronutrimentos fue $\mathrm{Fe}>\mathrm{Mn}>\mathrm{Zn}>\mathrm{Cu}=\mathrm{B}$.

Palabras clave: Musa AAA, banano, Cavendish, pH, aluminio, acidez intercambiable, magnesio.

\begin{abstract}
A hydroponic experiment was carried out with the objective of studying the effect of Al toxicity on the growth and absorption of water and nutrients by banana plants, and the effect of $\mathrm{Mg}$ and $\mathrm{pH}$ on $\mathrm{Al}$ toxicity. The six treatments evaluated consisted of the combination of four Mg rates (25, 50, 100, $200 \mathrm{mg} 1-1)$ and two Al rates (0 y $25 \mathrm{mg}^{-1^{-1}}$ ) at $3 \mathrm{pH}$ values which produced the following treatments: 1) $25-0 \mathrm{pH} 4$; 2) $25-25 \mathrm{pH} \mathrm{3}$; 3) $50-25 \mathrm{pH}$ 3 ; 4) $100-25 \mathrm{pH} 3$; 5) 200-25 pH 3; and 6) $25-25$ pH 5. Tissue culture plants of the Grand Naine clone between 7 and $10 \mathrm{~cm}$ height were grown in $20 \mathrm{~L}$ containers for 63 days. At the end of this time, plant height, pseudostem circumference, plant biomass, leaf emission rate, leaf area and nutrient absorption were measured. Al reduced the magnitude of all the growth variables, except leaf emission rate. The $\mathrm{Mg}$ rate of $50 \mathrm{mg} \mathrm{l}^{-1}$ partially reduced the effects of $\mathrm{Al}$ on plant height and pseudostem circumference. Greater concentrations of $\mathrm{Mg}$ gave similar results to those of $\mathrm{Mg}$ at $25 \mathrm{mg} \mathrm{l}^{-1}$. Aluminum affected biomass production principally that of the first order roots, the effect being partially reduced by the increment of $\mathrm{Mg}$ concentration in the solution. Water absorption significant-
\end{abstract}


ly decreased by $\mathrm{Al}$, while the $50 \mathrm{mg} \mathrm{l}^{-1} \mathrm{Mg}$ rate also tended to decreased water uptake. Increasing $\mathrm{pH}$ from 3 to 5reduced Al toxicity. Aluminum reduced biomass production by $65 \%$ compared to plants with no Al, while the total absorption of macronutrients decreased between 56 and $89 \%$ in the following order $\mathrm{Mg} \geq \mathrm{Ca}>\mathrm{S}>\mathrm{N} \geq \mathrm{K}>\mathrm{P}$ and micronutrients order of concentration reduction was $\mathrm{Fe}>\mathrm{Mn}>\mathrm{Zn}>\mathrm{Cu}=\mathrm{B}$ for the micronutrients.

Keywords: Musa AAA, bananas, Cavendish, pH, aluminum, exchangeable acidity, magnesium.

\section{Introducción}

La acidez y la escasa disponibilidad de nutrimentos son algunas de las razones de la baja productividad en los suelos (Baligar et al., 1998; Molina, 1998; Zapata, 2004). Se estima que los suelos ácidos ocupan alrededor de 3,95 billones de hectáreas, lo que corresponde a un $30 \%$ de la superficie cultivable del mundo (von Uexküll \& Mutert, 1995). Se estima, además, que el $35 \%$ de las tierras cultivables de América Central son ácidas reflejando el grado de degradación, meteorización y lixiviación que han experimentado (Baligar et al., 1998). Sin embargo, la acidificación de las tierras dedicadas al cultivo del banano en Costa Rica es causada principalmente por la utilización de fertilizantes de reacción ácida (Molina, 1998; Serrano, 2003). Al establecerse originalmente las plantaciones se seleccionaron tierras sin acidez, debido a que ésta es una característica no deseable cuando se escogen tierras para la siembra del banano (Jaramillo et al., 1979; Jaramillo \& Vázquez, 1900).

Los efectos adversos de la acidez sobre el crecimiento de los cultivos se deben a una serie de factores que incluyen bajo $\mathrm{pH}$, toxicidad de $\mathrm{Al}$ y/o $\mathrm{Mn}$ y deficiencias de P, Ca y Mg (Ayarza \& Salinas, 1982; Matsumoto, 2002). El Al y el Mn intercambiables son los principales elementos tóxicos en la mayoría de los suelos ácidos, el Al puede ocupar entre 4 y 94\% de la capacidad de intercambio catiónico (Matsumoto, 2002). La toxicidad del Al es el factor más perjudicial para las plantas en los suelos ácidos, particularmente cuando el pH es inferior de 5,5 (Bertsch, 1995). El valor de $\mathrm{pH}$ en el cual los niveles del Al alcanzan efectos perjudiciales es diferente entre especies y también entre variedades o cultivares de una misma especie (Tang et al., 1993; Van et al., 1997; Zhang \& Jessop, 1998), pero este valor depende también de ciertas condiciones del suelo como la mineralogía de las arcillas, el contenido de la materia orgánica, la presencia de otros cationes y aniones y de la salinidad total del suelo (Fassbender \& Bornemisza, 1987).
Experimentos en soluciones nutritivas han demostrado que concentraciones de Al que fluctúan entre 0,2 a $6,0 \mathrm{mg} \mathrm{l}^{-1}$ son suficientes para limitar el crecimiento de varias especies de plantas (Ayarza \& Salinas, 1982; Foy, 1984; Spain et al., 1975; Voight et al., 1999). Estos resultados se obtuvieron en experimentos con soluciones nutritivas y en suelo sobre agar en los cuales se añadieron varias concentraciones de Al. En general, los experimentos fueron diseñados para estimar el efecto de dosis de $\mathrm{Al}$ en períodos cortos. La reducción en la elongación de las raíces es el primer síntoma visible de la toxicidad del Al en muchas especies de plantas. El Al en concentraciones de micromoles en la solución inhibe fuertemente la división celular en las raíces y, en consecuencia, su crecimiento. Sin embargo, la tasa de reducción de la elongación disminuye en presencia de otros iones como el $\mathrm{Ca}$ y $\mathrm{Mg}$, debido a que la interacción de estos cationes con el $\mathrm{Al}$ reduce la toxicidad. La inhibición de la elongación, así como de la división de las células en la raíz, son consecuencia de la interacción del Al con las moléculas de ADN, lo que cambia la fluidez y la permeabilidad de las membranas celulares, reduce la actividad de la ATPasa ligada a las membranas, inhibe la absorción de $\mathrm{Ca}, \mathrm{Mg}$ y precipita los fosfatos (Cronan \& Grigal, 1995). Cambios de carga eléctrica en la superficie de la raíz y la presencia de otros iones, especialmente cationes, disminuyen la absorción del Al (Alva et al., 1986; Ayarza \& Salinas, 1982; Foy, 1984; Matsumoto, 2002; Ritchey et al., 1988). El Al absorbido por las plantas tiende a acumularse más en las raíces que en la parte aérea, variando la concentración según la especie. Se ha tratado de asociar la tolerancia $\mathrm{al} \mathrm{Al}^{+3}$ con la habilidad de las plantas para acumular o excluir el Al en la parte aérea, pero los resultados han sido contradictorios, lo que indica que la tolerancia es una característica propia de cada especie (Foy, 1984).

El uso de fertilizantes de reacción neta ácida (úrea, nitrato de amonio, sulfato de amonio, fosfato monoamónico y fosfato diamónico) contribuye a la acidificación del suelo de manera proporcional al índice de 
acidez fisiológica del fertilizante (Kass, 1996; Tisdale et al., 1993). El grado de acidificación de los suelos bananeros depende también del manejo y la edad de la plantación, en forma general, a mayor edad de la plantación mayor es la acidificación de los suelos. Se ha demostrado que el uso de fertilizantes de reacción ácida y la lixiviación de bases en el agua de drenaje de un suelo cultivado con banano durante un período de dos año redujeron el pH de 6,48 a 5,07 e incrementaron la acidez intercambiable de 0,07 a $1,16 \mathrm{cmol}_{(+)} 1^{-1}$ (Serrano, 2003). En Costa Rica existen 43.031 has cultivadas con banano (CORBANA, 2011) y es de esperarse que al menos el $70 \%$ de las tierras dedicadas al cultivo presenten algún grado de acidificación.

Considerando que las musáceas son de los principales cultivos de las zonas tropicales, existen pocos estudios acerca de la sensibilidad de estas plantas a la toxicidad del Al (Delvaux, 1995; Sharrock \& Frison, 1999; Wilson et al., 1987). La acidez reduce entre un 10 a $50 \%$ la cantidad de masa radicular en la planta de banano, dependiendo del nivel de acidificación de los suelos, en consecuencia, la productividad se puede reducir entre 50 a $67 \%$, si la acidez es el único factor limitante (Gaugel et al., 2003). Se ha observado que las plantas de banano acumulan Al en las raíces y cormo, mientras que la acumulación en la parte aérea es relativamente baja, lo que sugiere que la planta de banano no es tolerante al $\mathrm{Al}$ (Gaugel et al., 2003; Rufyikiri et al., 2000, 2001). Por otro lado, los plátanos pueden tolerar hasta un $77 \%$ de saturación de $\mathrm{Al}$ en el complejo de intercambio (Rodríguez-García et al., 1985). Investigación con varios cultivares de plátanos y un cultivar de banano (Gran Naine) demostró que una concentración de $78,5 \mathrm{uM}$ de $\mathrm{Al}$ redujo la absorción de agua entre 30 y $40 \%$ con respecto al testigo. La presencia de Al redujo también la absorción de $\mathrm{Ca}, \mathrm{Mg}, \mathrm{K}, \mathrm{N}_{-} \mathrm{NO}_{3} \mathrm{y}$ $\mathrm{N}-\mathrm{NH}_{4}$ y especialmente la de $\mathrm{Mg}$ que disminuyó en 80\% (Rufyikiri et al., 2000, 2001).

El incremento en la concentración de $\mathrm{Al}$ en el medio disminuye la absorción del $\mathrm{Mg}$ y el contenido de este nutrimento en diferentes partes de la planta. El efecto del $\mathrm{Al}$ en el crecimiento de la planta se puede prevenir con la presencia de altos niveles de $\mathrm{Mg}$ (alta relación $\mathrm{Mg} / \mathrm{Al}$ ) evitando así las deficiencias de $\mathrm{Mg}$ inducidas por la presencia del Al (Marschner, 1995). El Mg se encuentra en mayor proporción en las vacuolas, aunque también se encuentra abundantemente en el citosol donde ayuda a mantener el $\mathrm{pH}$ entre 6,5 y 7,5 , mientras que los cloroplastos contie- nen entre 10 y $20 \%$ del total de Mg en la hoja (Gil, 1995). Entonces, el incremento del Mg en la solución podría llegar a compensar los efectos tóxicos del Al en las plantas de banano (Gil, 1995; Marschner, 1995; Rufyikiri et al., 2001).

El objetivo del presente trabajo fue evaluar el efecto del $\mathrm{pH}$ y el $\mathrm{Mg}$ en la toxicidad del Al y en el crecimiento y absorción de nutrimentos y agua de plantas jóvenes de banano creciendo en una solución nutritiva.

\section{Materiales y métodos}

El experimento se condujo en el Centro de Investigación San Luis de la Compañía Bananera Atlántica (CISL-COBAL), en San Luis, distrito primero del cantón de Guácimo provincia de Limón, Costa Rica. El CISL está ubicado a $82 \mathrm{msnm}$, tiene una temperatura promedio anual de $27{ }^{\circ} \mathrm{C}$ y una precipitación anual promedio de $3.970 \mathrm{~mm}$. Se utilizaron plantas provenientes de cultivo de tejidos del clon "Gran Naine" de 7 a $10 \mathrm{~cm}$ de altura y con 4 a 5 hojas emitidas que tenían aproximadamente 4 semanas de edad y que estaban listas para iniciar la etapa de "endurecimiento". Las plantas se pusieron a crecer en potes de 20.01 de capacidad que contenían la solución nutritiva propuesta por Johnson y descrita por Lorenz \& Maynard (1988). El volumen de solución nutritiva en cada pote fue de 19.0 1. La concentración de nutrimentos $\left(\mathrm{mg} \mathrm{l}^{-1}\right)$ de la solución base fue: N: 104; P: 33; K: 138; Mg: 25; Ca 90; S: 35; Fe: 2,6; Cu: 0,1; Zn: 0,3; Mn 1; B 0,3 y Mo: 0,01 y se la cambió dos veces por semana. Se evaluaron los siguientes tratamientos: 1) 25 $\mathrm{mg} \mathrm{l}^{-1} \mathrm{de} \mathrm{Mg}+0 \mathrm{mg} \mathrm{l}^{-1} \mathrm{de} \mathrm{Al}, \mathrm{pH} 4$; 2) $25 \mathrm{mg} \mathrm{l}^{-1} \mathrm{de}$ $\mathrm{Mg}+25 \mathrm{mg} \mathrm{l}^{-1}$ de Al, $\mathrm{pH} \mathrm{3}$; 3) $50 \mathrm{mg} \mathrm{l}^{-1} \mathrm{de} \mathrm{Mg}+$ $25 \mathrm{mg}^{-1} \mathrm{l}^{-1} \mathrm{de} \mathrm{Al}, \mathrm{pH} 3$; 4) $100 \mathrm{mg} \mathrm{l}^{-1} \mathrm{de} \mathrm{Mg}+25 \mathrm{mg}$ $\mathrm{l}^{-1} \mathrm{de} \mathrm{Al}, \mathrm{pH} \mathrm{3}$; 5) $200 \mathrm{mg} \mathrm{l}^{-1} \mathrm{de} \mathrm{Mg}+25 \mathrm{mg} \mathrm{l}^{-1} \mathrm{de}$ $\mathrm{Al}, \mathrm{pH} 3$, y 6) $25 \mathrm{mg} \mathrm{l}^{-1} \mathrm{de} \mathrm{Mg}+25 \mathrm{mg} \mathrm{l}^{-1} \mathrm{de} \mathrm{Al}, \mathrm{pH}$ 5. Los tratamientos 1 y 2 sirvieron para evaluar el efecto del $\mathrm{Al}^{+3}$ a una misma concentración de $\mathrm{Mg}$, mientras que los tratamientos del 2 al 5 permitieron evaluar el efecto de las dosis de $\mathrm{Mg}$ sobre el $\mathrm{Al}^{+3}$ y los tratamientos 2 y 6 sirvieron para medir el efecto del $\mathrm{pH}$ sobre la toxicidad del $\mathrm{A}^{1+3}$. Para suplir el $\mathrm{Mg}$ adicional al suministrado en la solución nutritiva base (tratamientos 3, 4 y 5) se preparó una solución madre de $23.750 \mathrm{mg} \mathrm{l}^{-1}$ de $\mathrm{Mg}$ con $\mathrm{Mg}$ $\mathrm{SO}_{4} \cdot 7 \mathrm{H}_{2} \mathrm{O}$ y se tomaron alícuotas de 20,60 y 140 $\mathrm{ml}$ por cada pote con 191 de agua. El aporte de $\mathrm{Al}$ se hizo utilizando $\mathrm{Al}_{2}\left(\mathrm{SO}_{4}\right)^{3} \cdot 18 \mathrm{H}_{2} \mathrm{O}$, preparando una solución madre de $9.500 \mathrm{mg}^{-1} \mathrm{y}$ tomando 
alícuotas de $50 \mathrm{ml}$ por cada pote con 191 de agua. La concentración del $\mathrm{S}\left(\mathrm{mg} \mathrm{l}^{-1}\right)$ fue diferente en los distintos tratamientos por efecto del ión acompañante de las fuentes usadas para aportar el $\mathrm{Mg}$ y el Al, así 2 (79), 3 (112), 4 (177), 5 (307), 6 (79) con respecto a la indicada anteriormente en la solución base que fue la utilizada en el tratamiento 1 (35). Para obtener $\mathrm{pH} 5$ fue necesario sustituir el $\mathrm{KNO}_{3}$ de la solución Johnson por $\mathrm{K}_{2} \mathrm{CO}_{3}$ y $\mathrm{NH}_{4} \mathrm{NO}_{3}$ con la preparación de soluciones madre de $91.463 \mathrm{mg} \mathrm{l}^{-1}$ de $\mathrm{K}$ y de $31688 \mathrm{mg} \mathrm{l}^{-1}$ de $\mathrm{N}$ y se tomaron alícuotas de $20 \mathrm{ml}$ por pote. Se utilizó un diseño irrestricto al azar con 5 repeticiones, cada planta constituyó la unidad experimental respectiva. Al inicio y a las 9 semanas de iniciado el experimento se evaluaron las siguientes variables: 1) ritmo de emisión foliar (REF) utilizando la siguiente fórmula: $\mathrm{N}^{\circ} \mathrm{de}$ hojas totales emitidas / $\mathrm{N}^{\circ}$ de semanas evaluadas. Al momento de establecer el experimento se marcó la última hoja emitida completamente abierta y se tomó nota del estado de la hoja "candela", a partir de ahí se contaron las hojas emitidas al final de las 9 semanas; 2) índice de incremento en altura (IIA) que se logró midiendo desde la base del cormo hasta la "V" formada entre la última hoja completamente abierta y la hoja candela. El IIA se obtuvo a partir de la fórmula ( $\mathrm{cm}$ de altura final $-\mathrm{cm}$ altura inicial) / $\mathrm{N}^{\circ}$ de semanas evaluadas; 3) área foliar (AF) medida al inicio y final del experimento solamente en las hojas completamente abiertas. Se procedió a dibujar las hojas en papel, luego se recortó el dibujo por el perímetro y se determinó la masa del papel. El área se obtuvo al comparar la masa del dibujo de la hoja contra la masa de un dibujo del mismo tipo de papel de área conocida $\left(400 \mathrm{~cm}^{2}\right)$; 4) producción de biomasa (PB) determinada a las 9 semanas de iniciados los tratamientos. Se dividió la planta en raíz de segundo orden, raíz de primer orden, cormo, pseudo tallo y nervadura (incluye pseudo peciolo) de hojas inferiores y superiores; 5) lámina foliar (LF) de las hojas inferiores y superiores. La división de hojas superiores e inferiores fue arbitraria y se consideró el grupo de hojas superiores a las últimas 5 hojas emitidas (hojas más jóvenes) y el grupo de hojas inferiores a las restantes hojas al momento de la cosecha. Todos estos tejidos se secaron en estufa a $70{ }^{\circ} \mathrm{C}$ hasta alcanzar masa constante. Se calcularon los siguientes índices fisiológicos y parámetros morfológicos de acuerdo a las fórmulas respectivas descritas por Bertsch (1995), Rodríguez \& Leihner (2006) y Turner (1972): 1) índice de crecimiento absoluto (ICA) $\left.\left.=(m s f-m s i) / t_{2}-t_{p}\right)\right)$ donde $\mathrm{msf}=$ masa seca final, $\mathrm{msi}=$ masa seca inicial, $\mathrm{t}_{2}=$ tiempo final, $\mathrm{t}_{1}=$ tiempo inicial; 2) índice de crecimiento relativo $(\mathrm{ICR})=$ $\left((\ln m s f-\ln m s i) /\left(t_{2}-t_{l}\right)\right),(\ln =$ logaritmo natural $\left.) ; 3\right)$ índice de crecimiento foliar absoluto (ICFA): $\left(\left(a f_{2}\right.\right.$ $\left.\left.-a f_{p}\right) /\left(t_{2}-t_{p}\right)\right),\left(a_{2}=\right.$ área foliar final, af $=$ área foliar inicial); 4) índice de crecimiento foliar relativo (ICFR): $\left.\left(\left(\ln a f_{2}-\ln a f_{p}\right) /\left(t_{2}-t_{\nu}\right)\right) ; 5\right)$ tasa de asimilación neta (TAN): $\left(\left((m s f-m s i) x\left(\ln a f_{2}-\ln a f_{p}\right)\right) /\left(t_{2}-t_{p}\right)\right.$ $\left.x\left(a f_{2}-a f_{1}\right) ; 6\right)$ relación de área foliar (RAF): $\left(a f_{2} /\right.$ $m s f)$; 6) relación de masa foliar (RMF): (msfo/msf), $(\mathrm{msfo}=$ masa seca foliar $) ; 7)$ área foliar específica (AFE): (af $/ m s f o) ; 7)$ razón de masa radicular $(\mathrm{RMR}):(\mathrm{msr} / \mathrm{msf}),(\mathrm{msr}=$ masa seca de raíces); 8) relación de raíces a hojas $(\mathrm{RRH})$ : (msr/mofo) y; 9) relación de raíces a parte aérea (RRPA): ( $m s r /$ mspa $),($ mspa = masa seca parte aérea $)$.

Debido a que el método de determinación de la biomasa fue destructivo, la masa seca inicial se determinó de manera indirecta, tomando 10 plantas de iguales características a las utilizadas en el experimento en el mismo momento del establecimiento y se determinó la masa fresca y seca para calcular el porcentaje de masa seca por planta. En cada planta se determinó el peso fresco antes de colocarse en el pote respectivo y se asumió que las mismas presentaban igual porcentaje de masa seca que el promedio de las 10 plantas utilizadas en la determinación del porcentaje de masa seca, obteniendo así la masa seca inicial de cada una de las plantas usadas en cada tratamiento. Al momento de la cosecha se determinó la concentración de nutrimentos en los diferentes órganos de la planta. El análisis químico se realizó por el método de digestión húmeda (Westerman, 1990), el material seco de cada tejido de la planta fue molido y se hizo una digestión con ácido nítrico y se determinaron los contenidos de $\mathrm{P}, \mathrm{K}, \mathrm{Ca}, \mathrm{Mg}, \mathrm{S}, \mathrm{Fe}, \mathrm{Cu}, \mathrm{Zn}$, $\mathrm{Mn}$, y B por espectrometría de emisión de plasma, el $\mathrm{N}$ se determinó por el método de combustión en seco (Dumas) en analizador automático de $\mathrm{N}$ (Westerman, 1990). El análisis de la conductividad eléctrica se realizó por el método del puente eléctrico, utilizándose un conductímetro marca Beckman Instruments, Inc. Modelo RC-16C, el cual fue calibrado con una solución KCl 0,01M (Westerman, 1990). La absorción total de nutrimentos se calculó multiplicando la concentración de cada nutrimento por la masa seca de los diferentes componentes de la biomasa y se realizó la sumatoria para obtener la absorción total.

El consumo de agua se determinó aforando los potes a 19 litros con solución nutritiva, se determinó 
la masa del pote + solución nutritiva al inicio y final de cada ciclo de sustitución ( 3 y 4 días) y la diferencia de masa correspondió al consumo de agua en litros por la planta en dicho período. El consumo total de agua se obtuvo a partir de la sumatoria de agua consumida en cada ciclo de sustitución de la solución nutritiva. El pH de la solución se midió con el método del potenciómetro (Westerman, 1990) y se controló cada 24 horas y se ajustó siempre que la lectura presentara una desviación de $-0,17 \mathrm{y}+0,3$ de unidad de $\mathrm{pH}$ mediante la adición de $\mathrm{KOH} 0,5 \mathrm{M}$ y $\mathrm{HCl}$ concentrado, según fuera el caso.

Para el análisis estadístico se utilizó el modelo lineal general (GLM) procedimiento del programa S.A.S versión 8.1 (análisis de varianza) y pruebas de contraste. Se consideraron las diferencias significativas a valores de $p \leq 0,05$, la separación de medias se realizó por la prueba de diferencia mínima significativa.

\section{Resultados y discusión}

\subsection{Consumo total de agua}

El consumo de agua por las plantas de banano disminuyó significativamente con la aplicación de $\mathrm{Al}$ (T1 vs T2) (Figura 1). Resultados similares han sido reportados en otras especies (Wagatsuma 1983, Keltjens 1983, Tice 1992, Ishikawa \& Wagatsuma 1998, Matsumoto 2002). El menor consumo de agua por las plantas tratadas con $\mathrm{Al}$ fue el primer síntoma observado y las diferencias fueron estadísticamente significativas a partir del tercer día. Investigación conducida por Rufyikiri et al. (2001) encontró también que el primer síntoma que presentaron las plantas de banano y plátano tratadas con Al fue la disminución en el consumo de agua, las diferencias en banano Gran Naine fueron significativas a partir del día catorce de aplicado el Al. Es probable que las diferencias en el tiempo de detección del síntoma se deban a que las concentraciones de Al en la solución utilizada por Rufykiri y colaboradores fue menor por lo que la reducción en consumo de agua tardó más tiempo. En las plantas tratadas con Al no se encontraron cambios en el consumo de agua al incrementar las dosis de Mg hasta el día 38, momento que coincidió con el crecimiento más acelerado de las plantas. Un mayor consumo de agua sólo se presentó con la dosis de $50 \mathrm{mg} \mathrm{l}^{-1} \mathrm{de} \mathrm{Mg}$, consumo que descendió en las dosis superiores, debido al aumento de conductividad eléctrica de las dosis más altas de $\mathrm{Mg}$ (Figura 2) que ocasionó un menor consumo de agua por parte de la planta (De Oliveira et al., 2000). El incremento de $\mathrm{pH}$ de 3 a 5 fue más efectivo para mejorar la absorción de agua que el aumento en la dosis de Mg (Figura 1).

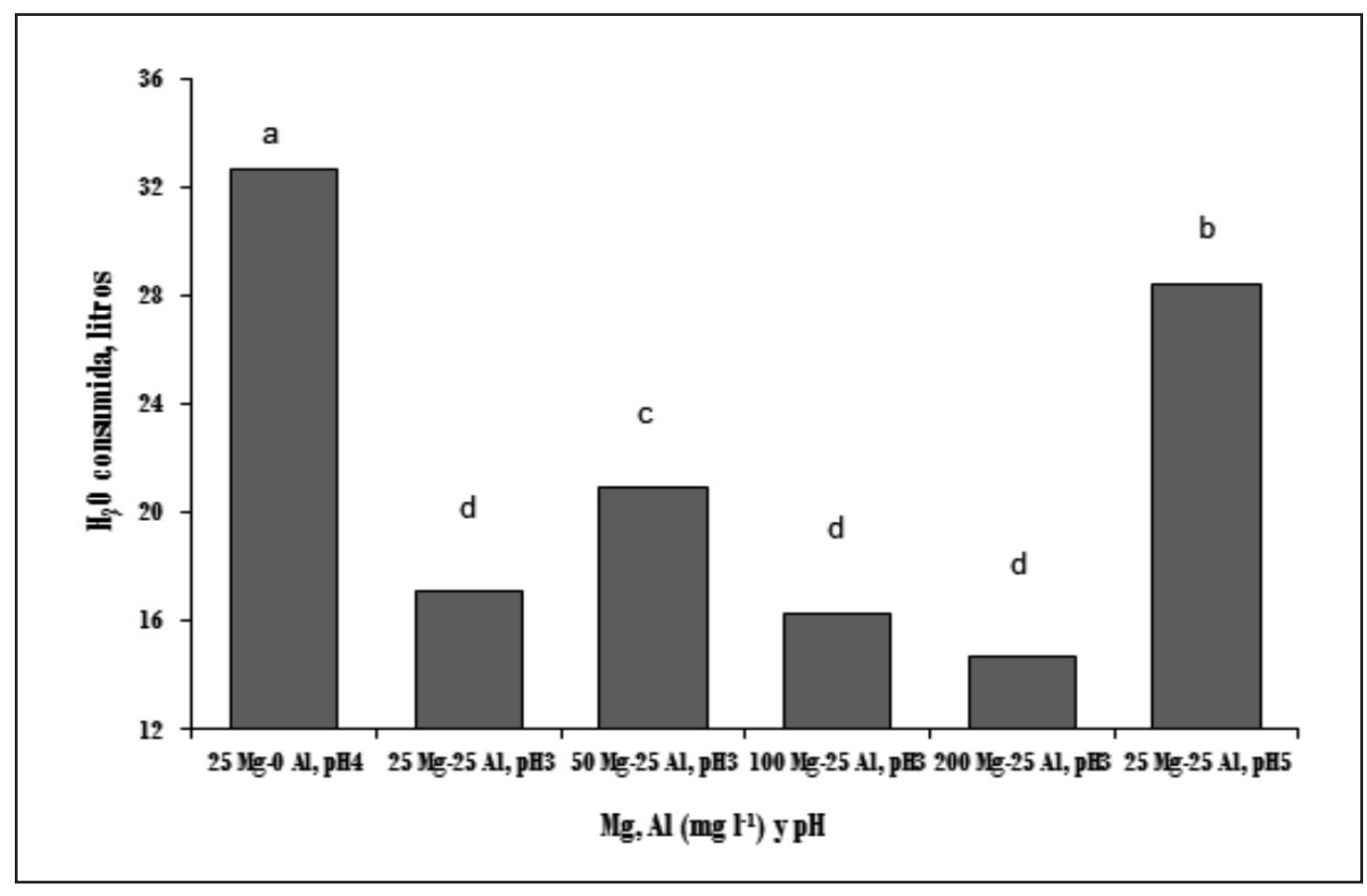

Figura 1. Consumo acumulado de agua a los 63 días de iniciado el experimento. 


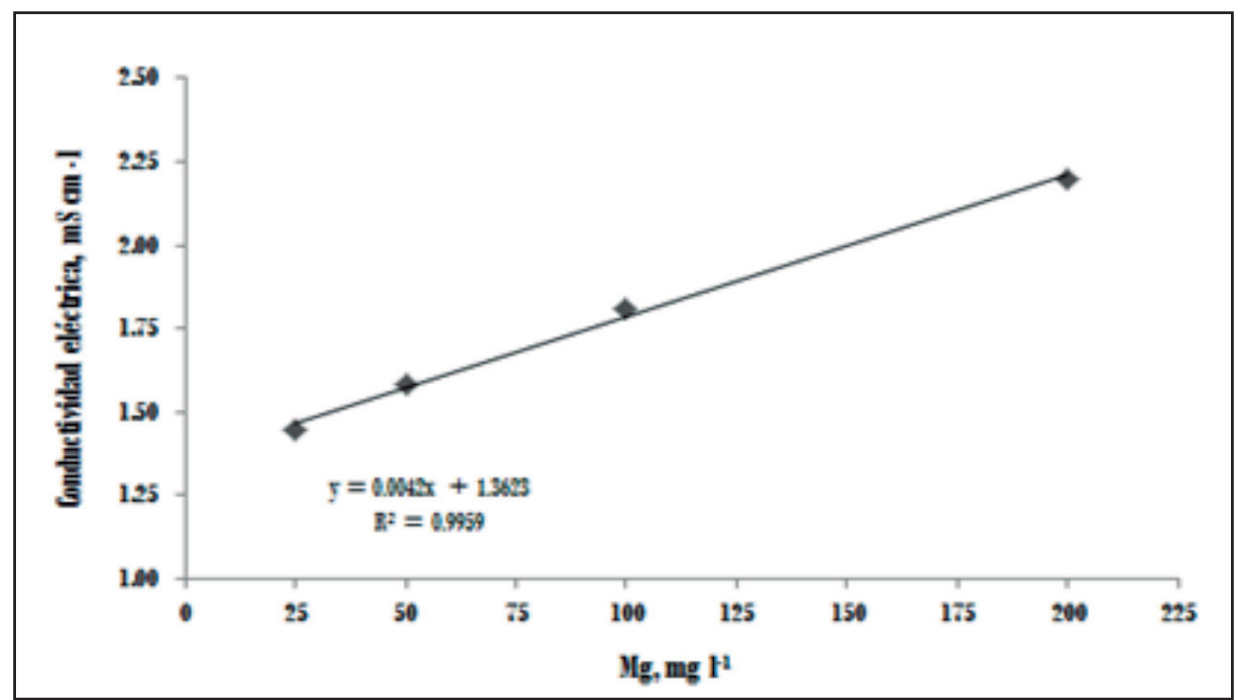

Figura 2. Conductividad eléctrica en función de la concentración de Mg en la solución nutritiva.

\subsection{Descripción de síntomas de toxicidad de Al en plantas de banano}

Los principales síntomas observados en las plantas creciendo en la solución nutritiva con Al con la menor dosis de $\mathrm{Mg}$ ( $\left.25 \mathrm{mg} \mathrm{l}^{-1}\right)$ fueron los siguientes: clorosis marcada a nivel de las hojas inferiores y reducción en el distanciamiento entre los pseudo pecíolos (Figura 3a). Como consecuencia de lo anterior, las plantas se tornaron más compactas (tipo roseta) y se observó una obstrucción foliar (arrepollamiento), lo que a su vez provocó una reducción de la altura en las plantas. De igual forma, las hojas de las plantas creciendo en solución nutritiva con $\mathrm{Al}$ fueron de menor tamaño y el pseudo tallo tuvo menor circunferencia. La reducción del tamaño de la hoja y de la distancia entre pseudo pecíolos es una respuesta del banano al déficit de agua (Turner, 1972). Síntomas similares fueron observados por Rufyikiri et al. (2001) quienes sugirieron que estos efectos pueden resultar de la reducción de crecimiento y de actividad radicular inducidos por la presencia de Al, condición que hace que la planta absorba menos agua.

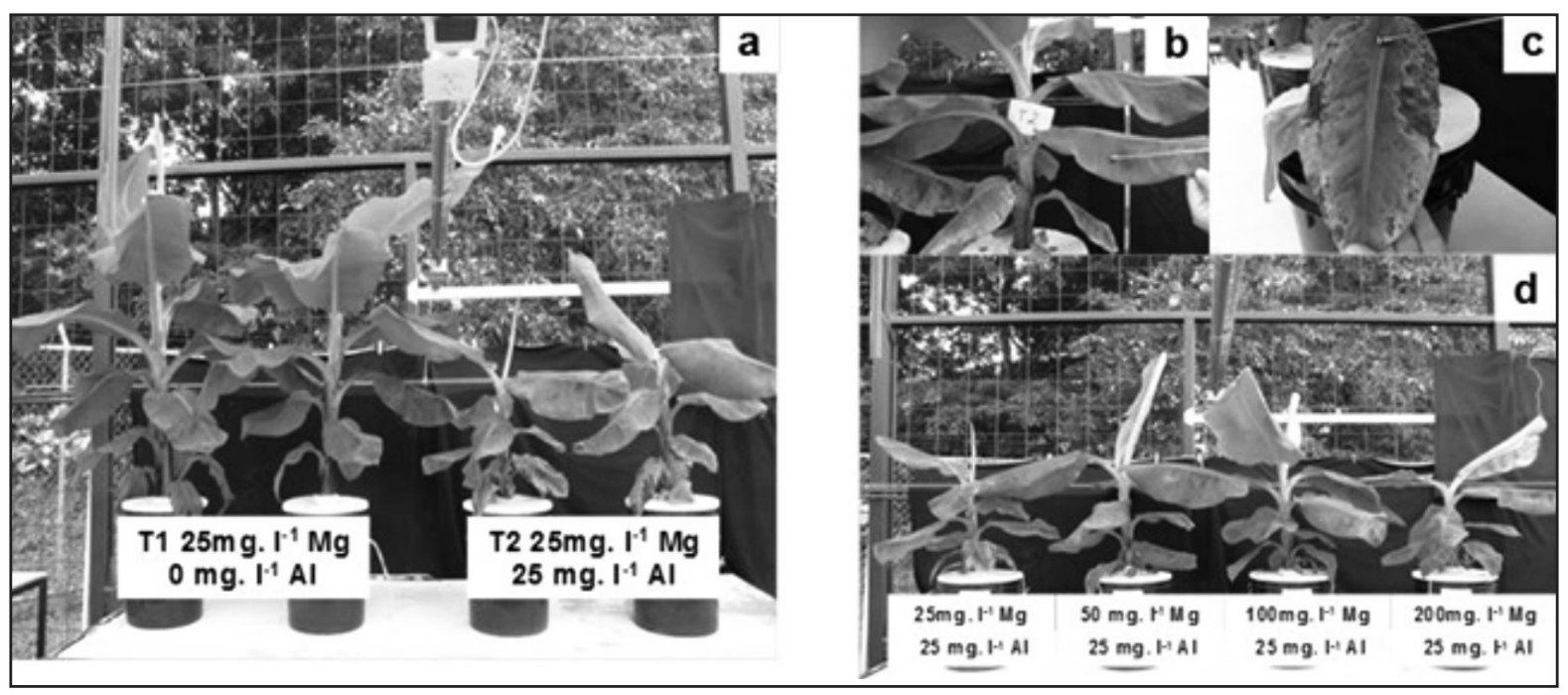

Figura 3. Efecto de la aplicación de Al en plantas de banano Gran Naine: (a) efecto de la toxicidad del Al en la reducción del distanciamiento entre pseudo peciolos; (b) inicio de clorosis; (c) necrosis avanzando hacia nervadura central; (d) efecto de concentración de $\mathrm{Mg}$ en la solución sobre la presencia de la clorosis. 
La clorosis se inició en los bordes de las hojas inferiores y fue ascendiendo a las hojas superiores (Figura 3b) avanzando luego hacia la nervadura central, pero ésta permaneció verde (Figura 3c), la lámina foliar se decoloró y se intensificó la clorosis con el paso del tiempo hasta que los tejidos se necrosaron. Esta sintomatología aparece por deficiencia de Mg (Tabla 1). El Mg es muy móvil en la planta, por esta razón la clorosis es ascendente (Villalobos \& Killorn, 2001). La clorosis en las hojas inferiores solo se presentó en las plantas que crecieron en la solución con la dosis menor de $\mathrm{Mg}$ a la que se aplicó Al (Figura 3d) lo que confirma el efecto positivo de la adición de cantidades mayores de $\mathrm{Mg}$ para evitar que se desarrolle la clorosis propia de los síntomas de carencia de Mg. Estos resultados demostraron que la clorosis fue inducida por la presencia del $\mathrm{Al}$ a través de una interferencia significativa de la absorción del $\mathrm{Mg}$ (Tabla 1). Los mismos síntomas fueron documentados por Rufyikiri et al. (2000) al evaluar el efecto del Al en plantas de banano y otros autores (Martin-prevel, 1980; Turner, 1972) han asociado también esta sintomatología a la deficiencia de Mg.

Tabla 1. Efecto del pH, la concentración del Mg en la solución nutritiva y el Al sobre la concentración de Mg en las diferentes partes de la planta analizadas.

\begin{tabular}{|c|c|c|c|c|c|c|c|c|c|c|c|}
\hline \multirow[b]{5}{*}{$\begin{array}{l}\text { Parte de } \\
\text { planta }\end{array}$} & \multicolumn{7}{|c|}{ TRATAMIENTOS } & \multicolumn{4}{|c|}{ Probabilidad $>$ F } \\
\hline & \multirow{3}{*}{$\begin{array}{c}\mathrm{Mg}, \mathrm{mg} \mathrm{l}^{-1} \\
\mathrm{Al}, \mathrm{mg} \mathrm{l}^{-1}\end{array}$} & 1 & 2 & 3 & 4 & 5 & 6 & \multirow{3}{*}{$\begin{array}{c}\text { Toxicidad } \\
\mathbf{A l}^{3+}\end{array}$} & \multirow{3}{*}{$\begin{array}{c}\text { Actividad } \\
\qquad \mathbf{A l}^{3+}\end{array}$} & \multirow{3}{*}{$\begin{array}{c}\text { Mg efecto } \\
\text { lineal }\end{array}$} & \multirow{3}{*}{$\begin{array}{l}\text { Mg efecto } \\
\text { cuadrático }\end{array}$} \\
\hline & & 25 & 25 & 50 & 100 & 200 & 25 & & & & \\
\hline & & $\mathbf{0}$ & 25 & 25 & 25 & 25 & 25 & & & & \\
\hline & pH & 4 & 3 & 3 & 3 & 3 & 5 & T1-T2 & T2-T6 & $\mathrm{T} 2,3,4,5$ & $\mathrm{~T} 2,3,4,5$ \\
\hline \multicolumn{12}{|c|}{ Concentración de $\mathrm{Mg}$ en los tejidos (\% de materia seca) } \\
\hline r.s.o. & & 0,55 a & $0,07 \mathrm{c}$ & $0,09 \mathrm{c}$ & $0,13 \mathrm{c}$ & $0,18 \mathrm{c}$ & $0,33 \mathrm{~b}$ & $<0,0001$ & 0,001 & $<0,0001$ & ns \\
\hline r.p.o. & & 0,26 a & $0,09 \mathrm{~d}$ & $0,12 \mathrm{~cd}$ & $0,15 \mathrm{c}$ & $0,20 \mathrm{~b}$ & $0,20 \mathrm{~b}$ & $<0,0001$ & $<0,0001$ & $<0,0001$ & 0,0880 \\
\hline Cormo & & $0,30 \mathrm{~b}$ & $0,12 \mathrm{~d}$ & $0,20 \mathrm{c}$ & $0,32 \mathrm{~b}$ & $0,42 \mathrm{a}$ & $0,22 \mathrm{c}$ & $<0,0001$ & $<0,0001$ & $<0,0001$ & 0,0003 \\
\hline ps. tallo & & $0,21 \mathrm{~b}$ & $0.08 \mathrm{~d}$ & $0,15 \mathrm{c}$ & $0,21 \mathrm{~b}$ & $0,30 \mathrm{a}$ & $0,14 \mathrm{c}$ & 0,0194 & ns & $<0,0001$ & 0,0003 \\
\hline $\begin{array}{l}\text { Ner. hojas } \\
\text { inf. }\end{array}$ & & $0,10 \mathrm{c}$ & $0,05 \mathrm{~d}$ & $0,12 \mathrm{c}$ & $0,24 \mathrm{~b}$ & $0,42 \mathrm{a}$ & $0,09 \mathrm{dc}$ & 0,0401 & 0,0918 & $<0,0001$ & ns \\
\hline $\begin{array}{l}\text { Ner. hojas } \\
\text { sup. }\end{array}$ & & $0,14 \mathrm{c}$ & $0,08 \mathrm{e}$ & $0,14 \mathrm{c}$ & $0,17 \mathrm{~b}$ & $0,23 \mathrm{a}$ & $0,10 \mathrm{dc}$ & $<0,0001$ & 0,0282 & $<0,0001$ & 0,0004 \\
\hline 1. hojas inf. & & $0,35 \mathrm{c}$ & $0,08 \mathrm{~d}$ & $0,32 \mathrm{c}$ & $0,75 \mathrm{~b}$ & $1,44 \mathrm{a}$ & $0,32 \mathrm{c}$ & $<0,0001$ & $<0,0001$ & $<0,0001$ & 0,0061 \\
\hline $\begin{array}{l}\text { 1. hojas } \\
\text { sup. }\end{array}$ & & $0,32 \mathrm{~d}$ & $0,15 \mathrm{f}$ & $0,36 \mathrm{c}$ & $0,51 \mathrm{~b}$ & $0,71 \mathrm{a}$ & $0,26 \mathrm{e}$ & $<0,0001$ & $<0,0001$ & $<0,0001$ & $<0,0001$ \\
\hline Planta total & & $0,271 \mathrm{c}$ & $0,108 \mathrm{e}$ & $0,225 \mathrm{~d}$ & $0,341 \mathrm{~b}$ & $0,530 \mathrm{a}$ & $0,214 \mathrm{~d}$ & $<0,0001$ & $<0,0001$ & $<0,0001$ & 0,0003 \\
\hline
\end{tabular}

r.s.o. (raiz de segundo orden); r.p.o. (raiz de primer orden), ps. (pseudo), nev. (nervadua), inf. (inferiores), sup.(superiores). Valores con una misma letra en cada fila no difieren estadísticamente según prueba de diferencia mínima significativa al $5 \%$; ns $=$ no significativo.

La concentración de $\mathrm{Mg}$ en las raíces disminuyó en presencia de $\mathrm{Al}$, aún en las dosis más altas de $\mathrm{Mg}$ (Tabla 1). La concentración de Mg en el cormo y pseudo tallo también disminuyó con la presencia del $\mathrm{Al}$ en el medio de crecimiento, pero se recuperó a partir de la dosis de $100 \mathrm{mg} \mathrm{l}^{-1}$ de $\mathrm{Mg}$. En el caso de las hojas y la planta total, el $\mathrm{Al}$ redujo la concentración de $\mathrm{Mg}$ en estos tejidos de forma significativa solo con la dosis más baja de $\mathrm{Mg}\left(25 \mathrm{mg} \mathrm{l}^{-1}\right)$, al subir la dosis de $\mathrm{Mg}$ se produjo una recuperación de la concentración de $\mathrm{Mg}$ en esos tejidos. Los valores de concentración del Mg a nivel foliar fueron normales y adecuados en los tratamientos 1
(Mg $25 \mathrm{mg} \mathrm{l}^{-1}, \mathrm{Al} 0 \mathrm{mg} \mathrm{l}^{-1} \mathrm{y} \mathrm{pH} \mathrm{4),3} \mathrm{(Mg} 50 \mathrm{mg} \mathrm{l}^{-1}, \mathrm{Al}$ $25 \mathrm{mg} \mathrm{l}^{-1}$ y pH 3) y $6\left(\mathrm{Mg}_{2} 5 \mathrm{mg} \mathrm{l}^{-1}, \mathrm{Al} 25 \mathrm{mg} \mathrm{l}^{-1} \mathrm{y} \mathrm{pH}^{2}\right)$, altos en los tratamientos $4\left(\mathrm{Mg} 100 \mathrm{mg} \mathrm{l}^{-1}, \mathrm{Al} 25 \mathrm{mg} \mathrm{l}^{-1}\right.$ y $\mathrm{pH} 3)$ y $5\left(\mathrm{Mg} 200 \mathrm{mg} \mathrm{l}^{-1}, \mathrm{Al} 25 \mathrm{mg} \mathrm{l}^{-1}\right.$ y $\left.\mathrm{pH} 3\right)$ y bajos en el tratamiento 2 ( $\left.\mathrm{Mg} 25 \mathrm{mg} \mathrm{l}^{-1}, \mathrm{Al} 25 \mathrm{mg} \mathrm{l}^{-1} \mathrm{y} \mathrm{pH} 3\right)$.

\subsection{Efecto de la concentración de $\mathrm{Mg} y$ del $\mathrm{pH}$ en las variables de crecimiento}

La aplicación de $\mathrm{Al}$ afectó en forma significativa las variables de crecimiento como acumulación de biomasa, altura de la planta, área foliar, circunferen- 
cia y número de hijos emitidos. El $\mathrm{Al}$ no tuvo efecto significativo en el número de hojas emitidas, ritmo de emisión foliar y el número de hojas al momento de finalizar el experimento (Tabla 2) coincidiendo con los resultados encontrados por Rufyikiri et al. (2000). Se ha demostrado que la emisión foliar y la tasa de crecimiento del fruto están gobernados principalmente por la temperatura ambiental (Robinson, 1996; Stover \& Simmonds, 1987; Turner \& Lahav, 1983).

Por otro lado, se observó un efecto favorable al incrementar el Mg de 25 a $50 \mathrm{mg} \mathrm{l}^{-1}$ en la altura de la planta y la circunferencia en la base del pseudo tallo, sin embargo, este incremento no fue suficiente para neutralizar el efecto del Al. Dosis superiores a $50 \mathrm{mg} \mathrm{l}^{1^{-1}}$ no tuvieron ningún efecto sobre la altura de la planta y la circunferencia del pseudo tallo debido al incremento en la conductividad eléctrica de la solución nutritiva al elevar la concentración de $\mathrm{Mg}$ (Figura 2), condición que afectó en forma negativa el crecimiento de la planta. La utilización de dosis mayores de $50 \mathrm{mg} \mathrm{l}^{-1}$ redujo el efecto positivo del incremento de Mg en la solución, lo que confirma que el banano es una planta susceptible a la salinidad (De Oliveira et al., 2000).

El incremento del $\mathrm{pH}$ de 3 a 5 fue más efectivo en disminuir la toxicidad del $\mathrm{Al}$ en las variables de crecimiento de la planta que el incremento de la dosis de $\mathrm{Mg}$ en la solución nutritiva (Tabla 2). Lo anterior se podría explicar por la presencia de las diferentes especies monoméricas de $\mathrm{Al}$ a diferente $\mathrm{pH}$, especialmente el $\mathrm{Al}^{+3}$ y el $\mathrm{Al}(\mathrm{OH})^{+2}$. Generalmente, el $\mathrm{Al}^{+3}$ es más fitotóxico que el $\mathrm{Al}(\mathrm{OH})^{+2}$ y esta especie es más tóxica que el $\mathrm{Al}(\mathrm{OH})_{2}{ }^{+}$, a pH 4 el $\mathrm{Al}$ está presente como $\mathrm{Al}^{+3}$, mientras que a pH 5 coexisten en equilibrio $\mathrm{Al}(\mathrm{OH})^{2+} \mathrm{y} \mathrm{Al}^{+3}$ (Miyasaka et al., 2007). Se considera que las dicotiledóneas son más sensitivas al $\mathrm{Al}(\mathrm{OH})_{2}^{+}$que al $\mathrm{Al}^{+3}$, mientras que para las monocotiledóneas lo son $\mathrm{al} \mathrm{Al}^{+3}$ posiblemente debido a que las monocotiledóneas tienen mayor capacidad de intercambio catiónico en la pared celular (Alva et al., 1986). Los resultados de este estudio sugieren que la planta de banano es afectada principalmente por $\mathrm{Al}^{+3}$ cuando el $\mathrm{pH}$ es igual o menor a 4,0.

Tabla 2. Efecto de la concentración del $\mathrm{Mg}$ y pH en la actividad del $\mathrm{Al}$ en las variables de crecimiento evaluadas.

\section{TRATAMIENTOS}

\begin{tabular}{|c|c|c|c|c|c|c|c|c|c|c|c|}
\hline & & 1 & 2 & 3 & 4 & 5 & 6 & \multirow[b]{2}{*}{$\begin{array}{c}\text { Toxicidad } \\
\mathbf{A l}^{3+}\end{array}$} & \multirow[b]{2}{*}{$\begin{array}{c}\text { Actividad } \\
\mathbf{A l}^{3+}\end{array}$} & \multirow[b]{2}{*}{$\begin{array}{c}\text { Mg } \\
\text { efecto } \\
\text { lineal }\end{array}$} & \multirow[b]{2}{*}{$\begin{array}{c}\text { Mg } \\
\text { efecto } \\
\text { cuadrático }\end{array}$} \\
\hline & $\begin{array}{l}\mathrm{Mg}, \mathrm{mgl}^{-1} \\
\mathrm{Al}, \mathrm{mg} \mathrm{l}^{-1}\end{array}$ & 25 & 25 & 25 & 25 & $\begin{array}{l}200 \\
25\end{array}$ & $\begin{array}{l}25 \\
25\end{array}$ & & & & \\
\hline Variables & $\mathbf{p H}$ & 4 & 3 & 3 & 3 & 3 & 5 & T1-T2 & T2-T6 & $\begin{array}{c}\text { T2, } \\
3,4,5\end{array}$ & $\begin{array}{c}\text { T2, } \\
3,4,5\end{array}$ \\
\hline m.s.f (g) & & $153,91 \mathrm{a}$ & $53,13 \mathrm{c}$ & $67,28 \mathrm{c}$ & $54,71 \mathrm{c}$ & $52,72 \mathrm{c}$ & $123,00 \mathrm{~b}$ & $<0,0001$ & $<0,0001$ & ns & ns \\
\hline a.f. (cm) & & 74,40 a & $41,33 \mathrm{~d}$ & $49,63 \mathrm{c}$ & $42,96 \mathrm{~cd}$ & $40,30 \mathrm{~d}$ & $60,60 \mathrm{~b}$ & $<0,0001$ & $<0,0001$ & ns & ns \\
\hline $\begin{array}{l}\text { r.e.f. (h } \\
\text { sem-1) }\end{array}$ & & $1,10 \mathrm{a}$ & $11,1 \mathrm{a}$ & $1,09 \mathrm{a}$ & $1,10 \mathrm{a}$ & $1,08 \mathrm{a}$ & $1,15 \mathrm{a}$ & ns & ns & ns & ns \\
\hline n.h.c. & & $9,44 \mathrm{a}$ & $9,15 \mathrm{a}$ & $9,00 \mathrm{a}$ & 9,32 a & $9,52 \mathrm{a}$ & $9,70 \mathrm{a}$ & ns & ns & ns & ns \\
\hline $\begin{array}{l}\text { Ar. Fol. } \\
(\mathrm{cm} 2)\end{array}$ & & $13.912 \mathrm{a}$ & $5.725 \mathrm{c}$ & $7.143 \mathrm{c}$ & $5.850 \mathrm{c}$ & $5.621 \mathrm{c}$ & $10.440 \mathrm{~b}$ & $<0,0001$ & ns & ns & ns \\
\hline $\begin{array}{l}\text { c.b. pt } \\
(\mathrm{cm})\end{array}$ & & $23,70 \mathrm{a}$ & $13,97 \mathrm{~d}$ & $16,38 \mathrm{c}$ & $15,14 \mathrm{~cd}$ & $14,80 \mathrm{~cd}$ & $21,28 \mathrm{~b}$ & $<0,0001$ & $<0,0001$ & ns & ns \\
\hline $\begin{array}{l}\text { n.b.e. } \\
\text { "hijos" }\end{array}$ & & $2,60 \mathrm{a}$ & $0,00 \mathrm{~h}$ & $0,75 \mathrm{~b}$ & $0,60 \mathrm{~b}$ & $0,60 \mathrm{~b}$ & $3,25 \mathrm{a}$ & $<0,0001$ & $<0,0001$ & ns & 0,0080 \\
\hline
\end{tabular}

Probabilidad $>$ F

m.s.f (masa seca final); a.f (altura final); r.e.f. (ritmo de emisión foliar, hojas por semana); n.h.c (número de hojas a cosecha); c.b. p.t. (circunferencia base del pseudo tallo); n.b.e (número de brotes emitidos). Valores con una misma letra en cada fila no difieren estadísticamente según prueba de diferencia mínima significativa al $5 \%$; $n s=$ no significativo. 
3.4. Efecto de la concentración de $\mathrm{Mg}$ y del $\mathrm{pH}$ en los índices de crecimiento y parámetros morfológicos

En general, el comportamiento de los índices de crecimiento estudiados fue la misma a la observada con los valores absolutos de las variables de crecimiento. $\mathrm{El} \mathrm{Al}$ afectó de manera negativa los índices fisiológicos IIA, ICA, ICR, ICFA, ICFR y TAN (Tabla 3). La dosis de $50 \mathrm{mg} \mathrm{l}^{-1}$ de $\mathrm{Mg}$ disminuyó los síntomas de toxicidad, pero solo hubo diferencias significativas entre tratamientos con el IIA. Las dosis más altas de $\mathrm{Mg}$ no logran mayor efecto compensatorio sobre la toxicidad del $\mathrm{Al}$, probablemente debido al efecto tóxico de la salinidad de la solución que fue causado por el incremento en las dosis de $\mathrm{Mg}$.

La magnitud de los parámetros RAF, RMF, RMR, RRH, RRPA se incrementó con la aplicación de Al (Tabla 3), a pesar de que con estos tratamientos se redujo el área foliar, la masa seca total (Tabla 2) y la masa seca en las diferentes partes en que se dividió la planta (Tabla 4), por esta razón, las relaciones calculadas tienden a aumentar a menor biomasa producida en las diferentes partes de la planta. No se encontraron diferencias entre las dosis de $\mathrm{Mg}$ evaluadas en los valores de RAF, RRH, RRPA, pero sí con respecto al tratamiento $\sin \mathrm{Al}$ y con $\mathrm{Al}$ a $\mathrm{pH} 5$ que fueron los que mayor biomasa produjeron.

\subsection{Efecto de la concentración de $\mathrm{Mg} y$ del $\mathrm{pH}$ en} la producción de biomasa en las diferentes partes de la planta

La presencia de $\mathrm{Al}$ en la solución afectó en forma negativa el desarrollo de las raíces, cormo, pseudo tallo, láminas foliares y nervaduras de hojas (Tabla 4). Hubo diferencias significativas con respecto al tratamiento sin Al. Las raíces de primer orden (primarias o raíces gruesas) fueron las más afectadas por el Al. El incremento en la dosis de $\mathrm{Mg}$ disminuyó levemente los efectos del $\mathrm{Al}$ en las raíces de primer orden produciendo mayor cantidad de biomasa, lo mismo ocurrió en el tratamiento con $\mathrm{Al}$ con la menor dosis de $\mathrm{Mg}$, pero a pH 5.

Tabla 3. Efecto de la concentración del $\mathrm{Mg}$ y pH en la actividad del Al en los índices fisiológicos y parámetros morfológicos evaluados.

\begin{tabular}{|c|c|c|c|c|c|c|c|c|c|c|c|}
\hline & & & & TRATAMI & ENTOS & & & & Probabil & idad $>$ F & \\
\hline & & 1 & 2 & 3 & 4 & 5 & 6 & & & & \\
\hline & $\begin{array}{l}\mathrm{Mg}, \\
\mathrm{mg} \mathrm{l}^{-1}\end{array}$ & 25 & 25 & 50 & 100 & 200 & 25 & $\begin{array}{c}\text { Toxicidad } \\
\mathbf{A l}^{3+}\end{array}$ & $\begin{array}{c}\text { Actividad } \\
\qquad \mathbf{A l}^{3+}\end{array}$ & $\begin{array}{c}\text { Mg } \\
\text { efecto }\end{array}$ & $\begin{array}{c}\mathrm{Mg} \\
\text { efecto }\end{array}$ \\
\hline & $\begin{array}{c}\text { Al, } \\
\text { mg l}^{-1}\end{array}$ & $\mathbf{0}$ & 25 & 25 & 25 & 25 & 25 & & & & \\
\hline Variables & pH & 4 & 3 & 3 & 3 & 3 & 5 & T1-T2 & T2-T6 & $\mathrm{T} 2, \mathbf{3 , 4 , 5}$ & $\mathrm{T} 2,3,4,5$ \\
\hline IIA $\left(\mathrm{cm} \mathrm{semana}{ }^{-1}\right)$ & & $6,82 \mathrm{a}$ & $3,78 \mathrm{~d}$ & $4,59 \mathrm{c}$ & $4,08 \mathrm{~cd}$ & $3,65 \mathrm{~d}$ & $5,90 \mathrm{~b}$ & $<0,0001$ & $<0,0001$ & ns & ns \\
\hline $\operatorname{ICA}\left(\mathrm{g}\right.$ semana $\left.^{-1}\right)$ & & $15,56 \mathrm{a}$ & $5,76 \mathrm{~b}$ & $7,19 \mathrm{~b}$ & $6,13 \mathrm{~b}$ & $5,62 \mathrm{~b}$ & $13,39 \mathrm{a}$ & $<0,0001$ & $<0,0001$ & ns & ns \\
\hline ICR $\left(\mathrm{g} \mathrm{g} \mathrm{semana}^{-1}\right)$ & & $0,58 \mathrm{a}$ & $0,51 \mathrm{bc}$ & $0,53 \mathrm{~b}$ & $0,53 \mathrm{~b}$ & $0,49 \mathrm{c}$ & $0,58 \mathrm{a}$ & 0,0005 & 0,0005 & ns & 0,0787 \\
\hline ICFA $\left(\mathrm{cm}^{2}\right.$ semana $\left.{ }^{-1}\right)$ & & $1401 \mathrm{a}$ & $614 b$ & $758 \mathrm{~b}$ & $645 \mathrm{~b}$ & $595 \mathrm{~b}$ & $1235 \mathrm{a}$ & $<0,0001$ & $<0,0001$ & ns & ns \\
\hline $\begin{array}{c}\operatorname{ICFR}\left(\mathrm{cm}^{2} \mathrm{~cm}^{2-1}\right. \\
\left.\text { semana }^{-1}\right)\end{array}$ & & $0,49 \mathrm{a}$ & $0,43 \mathrm{~b}$ & $0,46 \mathrm{ab}$ & $0,44 \mathrm{~b}$ & $0,44 \mathrm{~b}$ & $0,49 \mathrm{a}$ & 0,0035 & 0,0027 & ns & ns \\
\hline $\begin{array}{l}\text { TAN }\left(\mathrm{cm}^{2} \mathrm{~cm}^{2-1}\right. \\
\left.\text { semana }^{-1}\right)\end{array}$ & & $0,0055 \mathrm{a}$ & $0,0040 \mathrm{~b}$ & $0,0044 \mathrm{~b}$ & $0,0043 \mathrm{~b}$ & $0,0042 \mathrm{~b}$ & $0,0060 \mathrm{a}$ & 0,0113 & 0,0045 & ns & ns \\
\hline $\operatorname{RAF}\left(\mathrm{cm}^{2} \mathrm{~g}^{-1}\right)$ & & $90,57 \mathrm{bc}$ & $109,32 \mathrm{a}$ & $105,03 \mathrm{ab}$ & $105,39 a b$ & $105,39 \mathrm{ab}$ & $87,41 \mathrm{c}$ & 0,0379 & 0,0169 & ns & ns \\
\hline $\mathrm{RMF}\left(\mathrm{g} \mathrm{g}^{-1}\right)$ & & $0,49 \mathrm{c}$ & $0,54 \mathrm{a}$ & $0,50 \mathrm{c}$ & $0,51 \mathrm{bc}$ & $0,53 \mathrm{ab}$ & $0,54 \mathrm{a}$ & 0,0001 & $\mathrm{~ns}$ & ns & 0,0026 \\
\hline $\operatorname{AFE}\left(\mathrm{cm}^{2} \mathrm{~g}^{-1}\right)$ & & $184,61 \mathrm{ab}$ & $204,96 \mathrm{a}$ & $214,18 \mathrm{a}$ & $208,88 \mathrm{a}$ & $220,51 \mathrm{ab}$ & $164,42 \mathrm{~b}$ & ns & 0,0298 & ns & ns \\
\hline $\operatorname{RMR}\left(\mathrm{g} \mathrm{g}^{-1}\right)$ & & $0,14 \mathrm{c}$ & $0,20 \mathrm{ab}$ & $0,19 \mathrm{~b}$ & $0,22 \mathrm{ab}$ & $0,23 \mathrm{a}$ & $0,11 \mathrm{c}$ & 0.0030 & $<0,0001$ & ns & ns \\
\hline $\mathrm{RRH}\left(\mathrm{g} \mathrm{g}^{-1}\right)$ & & $0,28 \mathrm{~b}$ & $0,37 \mathrm{a}$ & $0,38 \mathrm{a}$ & $0,44 \mathrm{a}$ & $0,43 \mathrm{a}$ & $0,21 \mathrm{~b}$ & 0,0213 & 0,0002 & ns & ns \\
\hline $\operatorname{RRPA}\left(\mathrm{g} \mathrm{g}^{-1}\right)$ & & $0,16 \mathrm{~b}$ & $0,25 \mathrm{a}$ & $0,23 \mathrm{a}$ & 0,29 a & $0,29 \mathrm{a}$ & $0,13 \mathrm{~b}$ & 0,0048 & 0,0003 & ns & ns \\
\hline
\end{tabular}

IIA (indice de incremento de altura); ICA (indice de crecimiento absoluto); ICR (índice de crecimiento relativo); ICFA (indice de crecimiento foliar absoluto); ICFR (indice de crecimiento foliar relativo); TAN (tasa de asimilación neta); RAF (razón de área foliar); RMF (razón de masa foliar); $A F E$ (área foliar especifica); RMR (razón de masa radicular); $R R H$ (relación raíces a hojas); RRPA (relación raíces a parte aérea). Valores con una misma letra en cada fila no difieren estadísticamente según prueba de diferencia mínima significativa al $5 \%$; ns = no significativo. 
El incremento de las dosis de Mg no mejoró la producción de materia seca en el cormo y demás partes aéreas cuando se lo comparó con el tratamiento sin Al, sin embargo, el tratamiento con $\mathrm{Al}$ a $\mathrm{pH} 5$ y con la dosis menor de $\mathrm{Mg}$ sí redujo parcialmente los efectos tóxicos del $\mathrm{Al}$, alcanzando valores significativamente superiores al tratamiento con las misma dosis de $\mathrm{Mg}$, pero a $\mathrm{pH}$ 3. El incremento del $\mathrm{pH}$ de 3 a 5 con idéntica cantidad de Mg logró disminuir los efectos de la toxicidad del $\mathrm{Al}$ a nivel de biomasa producida en las raíces de segundo orden, nervadura y láminas inferiores, lo anterior posiblemente debido a una menor cantidad de $\mathrm{Al}^{+3}$ en la solución nutritiva en el tratamiento con $\mathrm{pH}$ 5, lo que probablemente redujo el efecto negativo del Al en la producción de biomasa aérea.

\subsection{Efecto de la concentración de Mgy del pH en la ab-} sorción total de nutrimentos por la planta de banano

Se observó que la aplicación de Al redujo la producción de biomasa (Tabla 4) lo cual hizo que la planta absorba solamente la tercera parte de N (Tabla 5). No se encontró efecto de las dosis crecientes de $\mathrm{Mg}$ sobre la producción de biomasa por parte de la planta (Tabla 5), razón por la cual no se afectó la absorción de N. El incremento de $\mathrm{pH}$ de 3 a 5 mejoró significativamente la absorción de $\mathrm{N}$, debido a que la concentración de $\mathrm{Al}^{+3}$ en la solución es menor a pH 5 por la coexistencia de $\mathrm{Al}^{+3}$ con $\mathrm{Al}(\mathrm{OH})^{2+}$ en la solución (Miyasaka et al., 2007), lo que reduce el efecto tóxico del Al. La absorción total del $\mathrm{P}$ disminuyó significativamente por la aplicación de $\mathrm{Al}$ (Tabla 5), situación que se explica por la menor biomasa producida por la planta cuando se adiciona $\mathrm{Al}$ (Tabla 4).

Tabla 4. Efecto de la concentración del $\mathrm{Mg}$ y del $\mathrm{pH}$ en la actividad del Al en la producción de biomasa en las diferentes partes de la planta.

TRATAMIENTOS

$\begin{array}{ccccccc} & 1 & 2 & 3 & 4 & 5 & 6 \\ \begin{array}{ccc}\mathrm{Mg}, \\ \mathrm{mg} \mathrm{l}^{-1}\end{array} & 25 & 25 & 50 & 100 & 200 & 25 \\ \begin{array}{c}\mathrm{Al}, \\ \mathrm{mg} \mathrm{l}^{-1}\end{array} & 0 & 25 & 25 & 25 & 25 & 25\end{array}$

\begin{tabular}{llllllllllllll} 
Variables & $\mathrm{pH}$ & 4 & 3 & 3 & 3 & 3 & 5 & T1-T2 & T2-T6 & T & T \\
\hline
\end{tabular}

Probabilidad $>$ F

$$
6
$$

5

xicidad Actividad efecto efecto $\mathbf{A l}^{3+} \quad \mathbf{A l}^{3+} \quad$ lineal cuadrático

Peso de la biomasa en las partes de la planta evaluadas $(\mathrm{g})$

\begin{tabular}{|c|c|c|c|c|c|c|c|c|c|c|}
\hline r.s.o. & 5,86 a & $3,75 \mathrm{~b}$ & $3,95 \mathrm{~b}$ & $4,00 \mathrm{~b}$ & $3,50 \mathrm{~b}$ & $6,08 \mathrm{a}$ & 0,0115 & 0,0060 & ns & ns \\
\hline r.p.o. & $15,36 \mathrm{a}$ & $5,38 \mathrm{c}$ & $9,18 \mathrm{~b}$ & $8,28 \mathrm{~b}$ & $8,40 \mathrm{~b}$ & $9,03 \mathrm{~b}$ & $<0,0001$ & 0,0146 & 0,0853 & 0,0352 \\
\hline Cormo & $16,46 \mathrm{a}$ & $4,39 \mathrm{c}$ & $5,42 \mathrm{c}$ & $3,54 \mathrm{c}$ & $3,52 \mathrm{c}$ & $12,84 \mathrm{~b}$ & $<0,0001$ & $<0,0001$ & 0,0078 & ns \\
\hline ps. tallo & $40,44 \mathrm{a}$ & $10,96 \mathrm{~cd}$ & $15,46 \mathrm{c}$ & $11,47 \mathrm{~cd}$ & $9,39 \mathrm{~d}$ & $28,44 \mathrm{~b}$ & $<0,0001$ & $<0,0001$ & 0,0149 & ns \\
\hline $\begin{array}{l}\text { Ner. hojas } \\
\text { inf. }\end{array}$ & $2,60 \mathrm{a}$ & $0,88 \mathrm{~b}$ & $8,83 \mathrm{~b}$ & $0,50 \mathrm{~b}$ & $0,62 \mathrm{~b}$ & $2,28 \mathrm{a}$ & $<0,0001$ & $<0,0001$ & 0,0594 & 0,0980 \\
\hline $\begin{array}{l}\text { Ner. hojas } \\
\text { sup. }\end{array}$ & $12,96 \mathrm{a}$ & $0,58 \mathrm{~b}$ & $5,93 \mathrm{~b}$ & $4,38 \mathrm{~b}$ & $4,26 \mathrm{~b}$ & $10,68 \mathrm{a}$ & 0,0089 & 0,0010 & ns & ns \\
\hline $\begin{array}{c}\text { 1. hojas } \\
\text { inf. }\end{array}$ & $12,06 \mathrm{a}$ & $0,48 \mathrm{~b}$ & $4,50 \mathrm{~b}$ & $4,00 \mathrm{~b}$ & $5,26 \mathrm{~b}$ & $14,48 \mathrm{a}$ & $<0,0001$ & $<0,0001$ & ns & ns \\
\hline $\begin{array}{c}\text { 1. hojas } \\
\text { sup. }\end{array}$ & $48,16 \mathrm{a}$ & $18,75 \mathrm{c}$ & $22,03 \mathrm{c}$ & $19,42 \mathrm{c}$ & $17,78 \mathrm{c}$ & $39,20 \mathrm{~b}$ & $<0,0001$ & $<0,0001$ & ns & ns \\
\hline $\begin{array}{c}\text { Planta } \\
\text { total }\end{array}$ & $153,91 \mathrm{a}$ & $53,13 \mathrm{c}$ & $67,28 \mathrm{c}$ & $55,59 \mathrm{c}$ & $52,72 \mathrm{c}$ & $123,00 \mathrm{~b}$ & $<0,0001$ & $<0,0001$ & ns & ns \\
\hline
\end{tabular}

r.s.o. (raiz de segundo orden); r.p.o. (raiz de primer orden), ps. (pseudo), nev. (nervadua), inf. (inferiores), sup.(superiores). Valores con una misma letra en cada fila no difieren estadísticamente según prueba de diferencia mínima significativa al $5 \%$; ns = no significativo. 
Tabla 5. Efecto de la concentración del $\mathrm{Mg}$ y $\mathrm{pH}$ en la actividad del Al en la absorción total de nutrimentos por la planta de banano.

\section{TRATAMIENTOS}

\begin{tabular}{|c|c|c|c|c|c|c|c|c|c|c|c|}
\hline \multirow[b]{4}{*}{ Nutrimentos } & \multirow{3}{*}{$\begin{array}{c}\mathrm{Mg}, \\
\mathrm{mg} \mathrm{l}^{-1} \\
\mathrm{Al}, \\
\mathrm{mg} \mathrm{l}^{-1}\end{array}$} & \multirow{2}{*}{$\begin{array}{l}1 \\
25\end{array}$} & 2 & 3 & 4 & 5 & \multicolumn{2}{|l|}{6} & \multirow{3}{*}{$\begin{array}{c}\text { Actividad } \\
\mathbf{A l}^{3^{+}}\end{array}$} & \multirow{3}{*}{$\begin{array}{l}\text { Mg } \\
\text { efecto } \\
\text { lineal }\end{array}$} & \multirow{3}{*}{$\begin{array}{c}\text { Mg } \\
\text { efecto } \\
\text { cuadrático }\end{array}$} \\
\hline & & & 25 & 50 & 100 & 200 & 25 & $\begin{array}{c}\text { Toxicidad } \\
\mathbf{A l}^{3+}\end{array}$ & & & \\
\hline & & 0 & 25 & 25 & 25 & 25 & 25 & & & & \\
\hline & pH & 4 & 3 & 3 & 3 & 3 & 5 & T1-T2 & T2-T6 & $\mathrm{T} 2,3,4,5$ & $\mathrm{~T} 2,3,4,5$ \\
\hline $\mathrm{N}(\mathrm{g})$ & & 6,46 a & $2,31 \mathrm{~b}$ & $2,86 \mathrm{~b}$ & $2,38 \mathrm{~b}$ & $2,14 \mathrm{~b}$ & $5,61 \mathrm{a}$ & $<0,0001$ & $<0,0001$ & ns & ns \\
\hline$P(g)$ & & $0,84 \mathrm{a}$ & $0,37 \mathrm{c}$ & $0,56 \mathrm{~b}$ & $0,52 \mathrm{bc}$ & $0,49 \mathrm{bc}$ & $0,59 \mathrm{~b}$ & $<0,0001$ & 0,0056 & ns & 0,0446 \\
\hline K (g) & & $12,16 \mathrm{a}$ & $4,66 \mathrm{~b}$ & $5,80 \mathrm{~b}$ & $4,42 \mathrm{~b}$ & $3,85 \mathrm{~b}$ & $6,00 \mathrm{a}$ & $<0,0001$ & $<0,0001$ & 0,0184 & ns \\
\hline $\mathrm{Ca}(\mathrm{g})$ & & $1,73 \mathrm{a}$ & $0,30 \mathrm{c}$ & $0,38 \mathrm{c}$ & $0,26 \mathrm{c}$ & $0,19 \mathrm{c}$ & $1,08 \mathrm{~b}$ & $<0,0001$ & $<0,0001$ & 0,0001 & ns \\
\hline $\operatorname{Mg}(\mathrm{g})$ & & 0,42 a & $0,06 \mathrm{~d}$ & $0,15 \mathrm{c}$ & $0,19 \mathrm{bc}$ & $0,28 \mathrm{~b}$ & $0,27 \mathrm{~b}$ & $<0,0001$ & $<0,0001$ & $<0,0001$ & 0,0071 \\
\hline $\mathrm{S}(\mathrm{g})$ & & $0,41 \mathrm{a}$ & $0,13 \mathrm{~d}$ & $0,20 \mathrm{~cd}$ & $0,18 \mathrm{~cd}$ & $0,25 \mathrm{bc}$ & $0,33 \mathrm{~b}$ & $<0,0001$ & $<0,0001$ & 0,0004 & ns \\
\hline $\mathrm{Fe}(\mathrm{mg})$ & & $452 \mathrm{a}$ & $48 \mathrm{~d}$ & $115 \mathrm{c}$ & $155 \mathrm{c}$ & $226 \mathrm{~b}$ & $52 \mathrm{~d}$ & $<0,0001$ & ns & $<0,0001$ & 0,0846 \\
\hline $\mathrm{Cu}(\mathrm{mg})$ & & $4,0 \mathrm{a}$ & $1,2 \mathrm{c}$ & $1,4 \mathrm{c}$ & $1,0 \mathrm{c}$ & $0,9 \mathrm{c}$ & $2,9 \mathrm{~b}$ & $<0,0001$ & $<0,0001$ & 0,0107 & ns \\
\hline $\mathrm{Zn}(\mathrm{mg})$ & & 14,2 a & $2,5 \mathrm{~b}$ & $2,9 \mathrm{~b}$ & $2,1 \mathrm{~b}$ & $1,9 \mathrm{~b}$ & $14,7 \mathrm{a}$ & $<0,0001$ & $<0,0001$ & 0,0107 & ns \\
\hline Mn (mg) & & $41,9 \mathrm{~b}$ & $6,5 \mathrm{c}$ & $6,9 \mathrm{c}$ & $4,7 \mathrm{c}$ & $5,5 \mathrm{c}$ & 78,2 a & $<0,0001$ & $<0,0001$ & 0,0051 & ns \\
\hline B (mg) & & 5,9 a & $1,8 \mathrm{c}$ & $3,4 \mathrm{~b}$ & $3,2 \mathrm{~b}$ & $5,0 \mathrm{a}$ & $3,3 \mathrm{~b}$ & $<0,0001$ & 0,0166 & $<0,0001$ & ns \\
\hline $\mathrm{Al}(\mathrm{mg})$ & & $22,6 \mathrm{e}$ & $155,8 \mathrm{~cd}$ & $280,3 \mathrm{a}$ & $261,2 \mathrm{ab}$ & $208,4 \mathrm{bc}$ & $111,2 \mathrm{~cd}$ & $<0,0001$ & ns & ns & 0,0042 \\
\hline
\end{tabular}

Valores con una misma letra en cada fila no difieren estadísticamente según prueba de diferencia mínima significativa al $5 \%$, ns = no significativo.

La presencia del Al redujo en más de $60 \%$ la absorción de K. El K asimilado por la planta fue similar para todas las dosis de $\mathrm{Mg}$ ya que produjeron la misma cantidad de biomasa. El incremento de $\mathrm{pH}$ de 3 a 5 mejoró significativamente la absorción del K (Marschner, 1995; Mengel \& Kirkby, 2000; Villalobos \& Killorn, 2001) debido a que este cambio mejoró el proceso de acumulación de biomasa. Se encontró efecto lineal negativo en la absorción de $\mathrm{K}$ al incrementar el contenido de $\mathrm{Mg}$ en solución, consecuencia del conocido antagonismo $\mathrm{Mg} / \mathrm{K}$ (Lacoeuilhe \& Martin-Prevel, 1971; Lahav \& Turner, 1992; Turner, 1972).
La presencia de Al redujo la absorción de Ca en 83\% debido a que el Al ocupa los sitios de absorción del Ca (Matsumoto, 2002). El incremento en la dosis de $\mathrm{Mg}$ de 25 a $50 \mathrm{mg} \mathrm{l}^{-1}$ mejoró levemente la absorción de $\mathrm{Ca}$, pero las diferencias observadas entre las diferentes dosis de $\mathrm{Mg}$ no fueron significativas. Se encontró un efecto lineal negativo entre el $\mathrm{Ca}$ absorbido y la concentración de $\mathrm{Mg}$ en solución que nuevamente se puede explicar por el efecto del antagonismo $\mathrm{Mg} / \mathrm{Ca}$ (Lacoeuilhe \& Martin-Prevel, 1971; Lahav \& Turner, 1992; Turner, 1972). El incremento de $\mathrm{pH}$ de 3 a 5 mejoró significativamente la absorción del $\mathrm{Ca}$, proba- 
blemente porque disminuyó la concentración de $\mathrm{Al}^{+3}$ en la solución nutritiva (Marschner, 1995; Mengel \& Kirkby, 2000; Villalobos \& Killorn, 2001).

La absorción de Mg disminuyó en $86 \%$ con la presencia de $\mathrm{Al}$ en la solución, debido probablemente a la competencia por los sitios de absorción y transportadores de la membrana celular (Bose et al., 2011; Rengel \& Robinson, 1989). La absorción de Mg se incrementó al aumentar la dosis de Mg en la solución, aunque no llegó a alcanzar la magnitud del Mg absorbido por las plantas del tratamiento sin $\mathrm{Al}$ (Tabla 5), lo anterior sugiere que existe fuerte competencia entre ambos elementos por los sitios de absorción y los transportadores de la membrana celular. Los cationes $\mathrm{Al}^{+3} \mathrm{y}$ $\mathrm{Mg}^{+2}$ tienen similar radio de hidratación $(0,48 \mathrm{~nm}$ para $\mathrm{Al}^{+3}$ y $0,428 \mathrm{~nm}$ para $\mathrm{Mg}^{+2}$ ) lo que favorece la competencia por los sitios de acople metal-enzima (Bose et al., 2011; Marschner, 1995; Matsumoto, 2002; Rengel \& Robinson, 1989). El Al redujo la absorción de $\mathrm{S}$ en $68 \%$ debido a la menor producción de materia seca. La absorción de S presentó un comportamiento similar a la del $\mathrm{Mg}$, debido a que la absorción mejora al incrementar la dosis Mg en solución, lo cual era de esperarse por al efecto del ión acompañante ya que la fuente de $\mathrm{Mg}$ utilizada fue sulfato de magnesio.

La absorción de $\mathrm{Fe}$ disminuyó casi en $90 \%$ al adicionar $\mathrm{Al}$ en la solución nutritiva, probablemente debido a la disminución en la producción de biomasa y al efecto de competencia por cargas en los sitios de absorción a nivel de raíces. Al incrementar las dosis de $\mathrm{Mg}$ aumentó la absorción de $\mathrm{Fe}$, aunque ésta fue inferior el valor absorbido por el tratamiento testigo sin $\mathrm{Al}$. $\mathrm{La}$ absorción de $\mathrm{Cu}, \mathrm{Zn}$ y $\mathrm{Mn}$ tuvo un comportamiento muy similar, la aplicación de $\mathrm{Al}$ redujo en forma significativa la absorción de estos nutrimentos, el incremento en la dosis de $\mathrm{Mg}$ disminuyó aún más la absorción de estos nutrimentos, probablemente debido al efecto antagónico del $\mathrm{Mg}$ con estos tres cationes.

\section{Referencias}

Alva, A., Edwords, D., Asher, C., \& Blamey, F. (1986). Relationships between root length of soybean and calculated activities of aluminium monomers in nutrient solutions. Soil Sci. Soc. Am. J., 50, 959-962.

Ayarza, M. A., \& Salinas, J. G. (1982). Estudio comparativo de la tolerancia al aluminio en tres leguminosas forrajeras. Suelos Ecuatoriales, 12(1), 110-126.

\section{Conclusiones}

La presencia de $\mathrm{Al}$ en la solución afectó la absorción de agua y el crecimiento de la planta de banano, situación que se reflejó en la reducción de la magnitud de las variables de crecimiento, siendo la acumulación de biomasa por las raíces de primer orden la más afectada. La utilización de Al causó síntomas de toxicidad que consistieron en la clorosis de hojas viejas y la necrosis de los bordes y ápices de hojas, las plantas se tornaron compactas $\mathrm{y}$ arrepolladas, tuvieron menor altura y acumularon menos biomasa. La dosis de $50 \mathrm{mg}^{-1}$ de $\mathrm{Mg}$ disminuyó parcialmente el efecto negativo de la toxicidad del $\mathrm{Al}^{+3}$ al mejorar en forma significativa la absorción de agua, la altura de planta y la circunferencia del pseudo tallo. Dosis mayores de Mg no mejoraron la expresión del efecto en estas variables con respecto a la dosis más baja, debido al incremento de la conductividad eléctrica de la solución nutritiva causado precisamente por las dosis más elevadas de $\mathrm{Mg}$ y S. El incremento del $\mathrm{pH}$ de 3 a 5 en presencia de Al fue más efectivo para reducir los efectos perjudiciales del $\mathrm{Al}$ que el aumento de las dosis de $\mathrm{Mg}$. Lo anterior debido probablemente a una menor concentración de $\mathrm{Al}^{+3}$ en solución, ya que a $\mathrm{pH} 3$ este elemento se encuentra en mayor proporción como $\mathrm{Al}^{+3}$ que es más reactivo, mientras que a $\mathrm{pH} 5$ existe un equilibrio entre $\mathrm{Al}^{+3} \mathrm{y}$ $\mathrm{Al}(\mathrm{OH})^{+2}$. La presencia del Al en la solución nutritiva redujo la absorción de todos los nutrimientos, el orden de reducción de los macronutrimentos fue $\mathrm{Mg} \geq \mathrm{Ca}>\mathrm{S}>\mathrm{N} \geq \mathrm{K}>\mathrm{P}$ y el de los micronutrimentos fue $\mathrm{Fe}>\mathrm{Mn}>\mathrm{Zn}>\mathrm{Cu}=\mathrm{B}$. Las dosis más altas de $\mathrm{Mg}$ mejoraron la absorción de $\mathrm{P}, \mathrm{Mg}, \mathrm{S}$, el $\mathrm{P}$ por el sinergismo P-Mg y el S por efecto del ión acompañante, pero disminuyeron la absorción del $\mathrm{Ca}$ y $\mathrm{K}$ por el antagonismo $\mathrm{Mg} / \mathrm{Ca}$ y $\mathrm{Mg} / \mathrm{K}$.

Baligar, V. C., Beaver, W. V., \& Ahlrichs, J. L. (1998). Nature and distribution of acid soils in the world. Paper presented at the Workshop to development a strategy for collaborative research and dissemination of technology in sustainable crop production in acid savannas and other problem soils of the world, Indiana, USA.

Bertsch, F. (1995). La fertilidad de los suelos y su manejo. San José, Costa Rica: ACCS. 
Bose, J., Babourina, O., \& Rengel, Z. (2011). Role of magnesium in alleviation of aluminium toxicity in plants. Journal of Experimental Botany, 62(7), 2251-2264.

CORBANA. (2011). Estadísticas de exportación bananera 2010 (pp. 734). San José, Costa Rica: CORBANA.

Cronan, C., \& Grigal, D. (1995). Use of calcium/aluminium ratios as indicators of stress in forest ecosystems. Journal of Environmental Quality, 24(2), 209-226. de Oliveira, S. L., Coelho, E. F., \& Borges, A. L. (2000). Irrigação e fertirrigação. In Z. J. Cordeiro (Ed.), Banana: produção, aspectos técnicos (pp. 60-72). Brasilia, Brasil: EMBRAPA Comunicação para Transferência de Tecnologia.

Delvaux, B. (1995). Soils. In S. Gowen (Ed.), Bananas and plantains (pp. 230-257). Londres, Inglaterra: Chapman \& Hall.

Fassbender, H., \& Bornemisza, E. (1987). Química de suelos con énfasis en suelos de América Latina ( $2^{\mathrm{da}}$ ed.). San José, Costa Rica: IICA.

Foy, C. D. (1984). Physiological effects of hydrogen, aluminium and manganese toxicities in acid soi1s. In F. Adams (Ed.), Soil acidity and liming ( $2^{\mathrm{da}}$ ed., pp. 57-97). Madison, Wisconsin: ASA.

Gaugel, C., Sierra, F., \& Arevalo, G. (2003). The problem of banana root deterioration and its impact on production: Latin American experience. Paper presented at the Simposio Internacional del Sistema Radical del Banano: hacia un mejor conocimiento para su manejo productivo, San José, Costa Rica.

Gil, F. (1995). Elementos de fisiología vegetal. Madrid, España: Mundi Prensa.

Jaramillo, R., Alvarado, A., Chaverri, B., \& Vázquez, A. (1979). Especificaciones teóricas para la determinación de la aptitud de las tierras para el cultivo del banano. Documento preliminar (pp. 7). San José, Costa Rica: ASBANA.

Jaramillo, R., \& Vázquez, A. (1900). Manual de procedimientos para la presentación y realización de estudios detallados de suelos y clasificación de tierras para el cultivo del banano. Edición revisada del Sistema de Clasificación para determinar la aptitud de las tierras para el cultivo del banano de Jaramillo, R. \& Vázquez, A. 1980 (pp. 25). San José, Costa Rica: Departamento de Investigaciones Agrícolas, CORBANA.

Kass, D. (1996). Fertilidad de suelos. San José, Costa Rica: EUNED.

Lacoeuilhe, J., \& Martin-Prevel, P. (1971). Culture sur milieu artificial. Carence en $\mathrm{K}, \mathrm{Ca}, \mathrm{Mg}$, chez le bananier : analyse foliaire. Fruits, 26(243-253).

Lahav, E., \& Turner, D. (1992). Fertilización del banano para rendimientos altos ( $2^{\mathrm{da}} \mathrm{ed}$.). Berna, Suiza: International Potash Institute.

Lorenz, O., \& Maynard, D. (1988). Knotts handbook for vegetable growers ( $3^{\text {rd }}$ ed.). New York, USA: Wiley.

Marschner, H. (1995). Mineral nutrition of higher plants $\left(2^{\text {nd }}\right.$ ed.). Londres, England: Academic Press.

Martin-prevel, P. (1980). La nutrition minerale du bananier dans le monde. Fruits, 35, 583-593.

Matsumoto, H. (2002). Plant Root Under Aluminium Stress: Toxicity and tolerance. In Y. Waisel, A. Eshel, \& U. Kafkafi (Eds.), Plant roots ( $3^{\text {rt }}$ ed., pp. 821-838). New York, USA: Hidden Half

Mengel, K., \& Kirkby, E. (2000). Principios de nutrición vegetal ( $4^{\text {ta }}$ ed.). Berna, Suiza: International Potash Institute.

Miyasaka, S. C., Hue, N. V., \& Dunn, M. A. (2007). Aluminium. In A. V. Baker \& D. J. Pilbeam (Eds.), Handbook on Plant Nutrition (pp. 439498). Florida, USA: CRC Press.

Molina, E. (1998). Encalado para la corrección de la acidez del suelo. San José, Costa Rica: ACCS.

Rengel, Z., \& Robinson, D. (1989). Competitive $\mathrm{Al}^{+3}$ inhibition of net $\mathrm{Mg}^{+2}$ uptake by intact Lolium multiflorum roots: I Kinetics. Plant physiology, 91, 1407-1413.

Ritchey, K., Sousa, D., \& Rodríguez, G. (1988). Testes biológicos para diagnóstico da deficiencia de calcio e toxicidade de alumínio em solos. Revista Brasilera de Ciencia do Solo, 12, 113-120.

Robinson, J. C. (1996). Bananas and plantains. Oxon, Inglaterra: W.K.Kellogg. 
Rodríguez-García, J., Rivera, E., \& Abruna, F. (1985). Crop response to soil acidity factors in ultisols and oxisols in Puerto Rico, 14 Plantains and Banana. Journal of Agricultural of University of Puerto Rico, 69, 377-382.

Rodríguez, W., \& Leihner, D. (2006). Análisis del crecimiento vegetal. In E. Villalobos (Ed.), Fisiología Vegetal (pp. 37). San José, Costa Rica: Editorial de la Universidad de Costa Rica.

Rufyikiri, G., Nootens, D., Dufey, E., \& Delvaux, B. (2000). Effect of aluminium on bananas (Musa ssp,) cultivated in acid solutions, Part I, Plant growth and chemical composition. Fruits, 55(6), 367-379.

Rufyikiri, G., Nootens, D., Dufey, E., \& Delvaux, B. (2001). Effect of aluminium on bananas (Musa ssp,) cultivated in acid solutions, Part II, Water and Nutrient uptake. Fruits, 56(6), 5-16.

Serrano, E. (2003). Acidificación de los suelos bananeros de Costa Rica y su relación con la toxicidad de aluminio en las raices del cultivo de banano. Paper presented at the Simposio Internacional del Sistema Radical del Banano: hacia un mejor conocimiento para su manejo productivo, San José, Costa Rica.

Sharrock, S., \& Frison, E. (1999). Musa production around the world - varieties and regional importante. Networking banana and plantain. In INIBAP (Ed.), INIBAP annual report 1998 (pp. 42-47). Montpellier, Francia: INIBAP, International Network for the Improvement of Banana and Plantain.

Spain, J., Francis, C., Howeler, R., \& Calvo, F. (1975). Differential species and varietal tolerance to soil acidity in tropical crops and pastures. In E. Bornemisza \& A. Alvarado (Eds.), Soil management in tropical America (pp. 308-329). Raleigh, USA: North Caroline State University.

Stover, R. H., \& Simmonds, N. W. (1987). Bananas ( $3^{\text {rd }}$ ed.). New York, USA: Wiley.

Tang, H., Houben, V., Nzok Mbouti, C., \& Dufey, E. (1993). Diagnostic précoce de la résistance de cultivars de riz (Oriza sativa L,) á la toxicité aluminique. Agronomie, 13, 853-860.

Tisdale, S., Nelson, W., Beaton, J., \& Havlin, J. (1993). Soil fertility and fertilizers $\left(5^{\text {th }}\right.$ ed.). Columbus, USA: Mc Millan.
Turner, D. (1972). Banana plant growth. 1. Gross morphology. 2. Dry matter production leaf area and growth analysis. Australian Journal Experimental Agric Anim. Husb., 12(209-224).

Turner, D., \& Lahav, E. (1983). The growth of banana plants in relation to temperature. Australian Journal Plant Physiology, 10(1), 43-53.

Van, J., Costa de Macedo, C., Kinet, J. M., \& Bouharmont, J. (1997). Selection of Al-resistant plants from a sensitive rice cultivar, using somaclonal variation in vitro and hydroponic cultures. Euphytica, 97, 303 -310.

Villalobos, E., \& Killorn, R. (2001). Nutrición mineral. In E. Villalobos (Ed.), Fisiología de la producción de los cultivos tropicales (pp. 161196.). San José, Costa Rica: Editorial Universidad de Costa Rica.

Voight, P. W., Godwin, H. W., \& Morris, D. R. (1999). Effect of four acid soils on root growth of clover seedlings using a soil-on-agar procedure. Plant and Soil, 205, 51-56.

von Uexküll, H., \& Mutert, E. (1995). Global extent, development and economic impact of acid soi1s. Plant and Soil, 171, 1-15.

Westerman, R. L. (1990). Soil testingand plant analysis ( ${ }^{\text {rd }}$ ed.). Madison, Wisconsin, USA: Soil Science Society of America.

Wilson, G. F., Swenenen, R., \& de Langhe, E. (1987). Effects of mulch and fertilizer on yield and longevity of a medium and giant plantain and a banana cultivar. Paper presented at the Proceedings of the third meeting. International Cooperation for effective plantain and banana research, Abidjan, Costa de Marfil.

Zapata, R. (2004). Química de la acidez del suelo. Medellín, Colombia: Universidad Nacional de Colombia.

Zhang, X., \& Jessop, R. S. (1998). Analysis of genetic variability of aluminium tolerance response in triticale. Euphytica, 102, 177-182. 